\title{
SEMI-STABLE STOCHASTIC PROCESSES $\left({ }^{(}\right)$
}

\author{
BY \\ JOHN LAMPERTI
}

0. Introduction. An interesting chapter in modern probability theory began with the search for all the possible limit distributions for sums of independent, identically-distributed random variables. The result - the theory of the stable laws (see, for instance, [1] or [6]) - generalizes and illuminates the original examples of normal convergence with which the problem originated. The purpose of this paper is to formalize and study an analogous situation in the theory of stochastic processes.

To introduce the problem we use an example which has been very well known for a long time. Let $\left\{X_{t}\right\}$ be a simple random walk on the line in which a moving particle starts from 0 at $t=0$ and makes transitions at times $t=n \tau, n$ an integer. The transitions are moves through a distance $\delta$ to the right or left, each with probability $1 / 2$. Then if $\tau \rightarrow 0$ and $\delta \rightarrow 0$, but $\delta^{2} / \tau \rightarrow 1$, the process $\left\{X_{t}\right\}$ converges to the Brownian motion (Wiener) process in the sense that the joint distribution of $\left(X_{t_{1}}, \cdots, X_{t_{k}}\right)$ for the random walks converges to that for Brownian motion for all finite $t$-sets $\left({ }^{2}\right)$. It is quite natural to raise the following question: Which processes can similarly occur as limits upon subjecting a fixed stochastic process to infinite contractions of its time and space scales? It is essentially this class which we call "semi-stable." The name is intended to suggest the analogy with the theory of stable laws, and is rendered more appropriate by the fact (see $\$ 2$, Example 1 below) that a semi-stable process, if it is assumed to have independent increments, must actually be a stable one $\left({ }^{3}\right)$.

1. Foundations. As suggested by footnote 2, we shall consider two stochastic processes $\left\{x_{t}\right\}$ and $\left\{y_{t}\right\}$ to be the same if they have the same state space and finite-dimensional distribution functions; we indicate this by writing $\left\{x_{t}\right\} \approx\left\{y_{t}\right\}$. All the processes considered have states in Euclidean space of $s$ dimensions, non-negative time parameter, and we assume throughout the continuity condition

Received by the editors August 2, 1961.

(1) This work was supported in part by National Science Foundation Grant Number 9669 at Stanford University.

(2) Actually there is convergence in the more powerful "weak" or "invariance principle" sense which entails limit distributions for many path-functionals. In this paper, however, we shall consider only convergence of finite-dimensional distributions.

(3) In [6] there is a definition of "semi-stable laws"; this definition seems no longer to be in common use and is not related to the way in which the term "semi-stable" is used in this paper. 


$$
\lim _{h \rightarrow 0} \operatorname{Pr}\left(\left\|x_{t+h}-x_{t}\right\|>\epsilon\right)=0
$$

for every $t \geqq 0, \epsilon>0$. We shall speak of a "proper" process if $x_{t}$ has a nondegenerate distribution for every $t>0$.

Definition 1. Two processes $\left\{x_{t}\right\}$ and $\left\{y_{t}\right\}$ belong to the same type (denoted $\left\{x_{t}\right\} \sim\left\{y_{t}\right\}$ ) if there exist constants $b, c$, respectively a positive number and an s-vector, such that $\left\{x_{t}\right\} \approx\left\{b y_{t}+c\right\}$.

It is easily verified that this is an equivalence relation, and that the Markov property, stationary transition probabilities, and the continuity condition (1) hold either for all processes of a given type or for none.

Definition 2. A process $\left\{x_{t}\right\}$ is "semi-stable" if it obeys the condition (1) and if for every $a>0,\left\{x_{a t}\right\} \sim\left\{x_{t}\right\}$.

The semi-stable property can be rephrased by saying there exist functions $b(a)$ with positive real values, and $c(a)$ with values in $R_{\mathbf{s}}$, such that for every $a>0,\left\{x_{a t}\right\}$ and $\left\{b(a) x_{t}+c(a)\right\}$ are the same process. This property also holds for all or none of the members of a given type, so that one can speak of a semi-stable type of processes. The first theorem, proved below in $\$ 3$, illuminates these notions somewhat.

THEOREM 1. If $\left\{x_{t}\right\}$ is a proper semi-stable process, then

$$
b(a)=a^{\alpha} \text { for some } \alpha \geqq 0 .
$$

If $\alpha \neq 0$, the distribution of $x_{0}$ is concentrated at a point $\omega$ and

$$
c(a)=\omega\left(1-a^{\alpha}\right) .
$$

If $\alpha=0$, then $c(a) \equiv 0$ and the process is trivial in the sense that $x_{t}=x_{0}$ a.s. for each $t$.

REMARKs. The constant $\alpha$ is easily seen to be the same for all processes of the same type; we accordingly can and will speak of a semi-stable process or type of order $\alpha$. We observe that each nontrivial semi-stable type of order $\alpha$ contains a process with $x_{0}=0$ and such that $\left\{x_{a t}\right\} \approx\left\{a^{\alpha} x_{t}\right\}$. Thus in trying to find or classify semi-stable processes these assumptions may be imposed without loss of generality. It is also easy to establish a one-to-one correspondence between the processes of one order and those of another; in fact, if $\left\{x_{t}\right\}$ is semi-stable of order $\alpha>0$, and $x_{0}=0$, then the process $\left\{y_{t}\right\}$ defined by

$$
y_{t}=\left\|x_{t}\right\|^{\beta / \alpha-1} x_{t}
$$

is semi-stable of order $\beta>0$. The proof is obvious and will be omitted.

The point of all this is that the semi-stable processes defined above are the only processes which can result from certain limiting operations. Let $\left\{X_{t}\right\}$ be a discrete or continuous time $\left({ }^{4}\right), s$-dimensional stochastic process.

(4) If $\left\{X_{t}\right\}$ is discrete parameter, we shall speak of the random variable $X_{t}$ meaning really $X_{[t] \text {. }}$ 
As suggested by the procedure used in the example in the introduction, we next assume that there exist real and vector valued functions $0<f(\xi) \nearrow \infty$, $g(\xi)$ and a proper $s$-dimensional process $\left\{x_{t}\right\}$ satisfying assumption (1) such that

$$
\lim _{\xi \rightarrow \infty}\left\{\frac{X_{\xi t}+g(\xi)}{f(\xi)}\right\}=\left\{x_{t}\right\}
$$

in the sense of convergence of finite-dimensional joint distributions. Our main result is then contained in

Theorem 2. If (5) holds, the process $\left\{x_{t}\right\}$ is semi-stable, and

$$
f(\xi)=\xi^{\alpha} L(\xi), \quad g(\xi)=\omega(\xi) \xi^{\alpha} L(\xi)
$$

where $\alpha>0, L(\xi)$ is a slowly varying function $\left({ }^{5}\right)$, and the vector valued function $\omega(\xi)$ has a limit $\omega$ as $\xi \rightarrow \infty$. The order of $\left\{x_{t}\right\}$ is then $\alpha$, and $x_{0}=\omega$. Conversely, every semi-stable process of positive order is such a limit for some process $\left\{X_{t}\right\}$.

This is also proved in $\S 3$. We can now pose two obvious problems: (i) "find" (or classify, or construct) all semi-stable processes, and (ii) determine their "domains of attraction." (We say that $\left\{X_{t}\right\}$ belongs to the domain of attraction of $\left\{x_{t}\right\}$ when (5) holds.) Problem (i) has a simple solution, which is not altogether satisfying but should be mentioned. It is as follows: if $\left\{y_{\tau}\right\}$ is a strictly stationary process, $-\infty<\tau<\infty$, continuous in the sense (1), and if for some $\alpha>0$

$$
x_{t}=t^{\alpha} y_{\log t} \quad \text { for } t>0, \quad x_{0}=0,
$$

then $\left\{x_{t}\right\}$ is semi-stable of order $\alpha$. Conversely, every nontrivial semi-stable process with $x_{0}=0$ is obtained in this way from some stationary process $\left\{y_{\tau}\right\}$. The proof simply consists of noticing that if $\left\{y_{\tau}\right\}$ is stationary, then

$$
\left\{x_{a t}\right\} \approx\left\{a^{\alpha} t^{\alpha} y_{\log t+\log a}\right\} \approx\left\{a^{\alpha} t^{\alpha} y_{\log t}\right\} \approx\left\{a^{\alpha} x_{t}\right\}
$$

while if $\left\{x_{a t}\right\} \approx\left\{a^{\alpha} x_{t}\right\}$, then

$$
\left\{y_{\tau+\sigma}\right\} \approx\left\{e^{-\alpha \tau} e^{-\alpha \sigma} x_{e^{\tau} e^{\sigma}}\right\} \approx\left\{e^{-\alpha \tau} x_{e^{\tau}}\right\} \approx\left\{y_{\tau}\right\} .
$$

One instance of $(7)$ has been used by Doob to deduce properties of the (stationary) Ornstein-Uhlenbeck velocity process from those of the Wiener process.

The unattractive feature of this solution to problem (i) is that the most interesting class of semi-stable processes has not been identified-the Markov processes with stationary transition probabilities. Correspondence (7) does preserve the Markov property but not stationarity. In the example of Doob mentioned above, for instance, only $\alpha=1 / 2$ gives an $\left\{x_{t}\right\}$ with stationary

(5) That is, $L(\xi)$ is positive and satisfies $L(c \xi) / L(\xi) \rightarrow 1$ as $\xi \rightarrow \infty$ for any $c>0$ (see [3]). 
transition probabilities, although $\left\{x_{t}\right\}$ is semi-stable and Markov for all values of $\alpha>0$. (See $\$ 4$.) In the next section we concentrate upon $\left\{x_{t}\right\}$ which are stationary Markov processes, and present some examples and results which fall short of finding all such processes.

The second question above, concerning "domains of attraction," seems even further from a complete solution. For instance, all of the work on conditions under which the central limit theorem is valid for Markov processes, martingales, stationary processes, etc., is pertinent to determining the "domain of attraction" of the Brownian motion process in our sense.

One further remark may be in order. It is natural to ask whether there is not an analogue of the infinitely-divisible laws in the same spirit in which semi-stable processes are analogous to stable laws. Some reflection has led me to the conclusion that the "correct" analogue is simply the class of all continuous-parameter processes which are continuous in the sense (1), so no interesting new class arises in this way.

2. Examples of semi-stable processes. In this section it will be assumed that all processes considered are Markovian and have stationary transition probabilities; also $\alpha>0$ and $x_{0}=0$.

EXAMPLE 1. Independent increments. A remark about terminology is first called for. In [6], P. Levy defines a "stable" distribution function as one such that if $X_{1}$ and $X_{2}$ are independent random variables with the given distribution, then for every $a_{1}>0, a_{2}>0$ there exists $a_{3}>0$ satisfying

$$
a_{1} X_{1}+a_{2} X_{2}=a_{3} X_{3}
$$

where $X_{3}$ again has the given distribution. Levy calls the distribution "quasistable" if (8) holds with a constant (depending on $a_{1}$ and $a_{2}$ ) added to the right-hand side. This distinction is not usually maintained today, and all of these laws are simply called "stable." However, we will now show that $a$ process $\left\{x_{t}\right\}$ with stationary independent increments and with $x_{0}=0$ is semistable if and only if the increments have distributions which are stable in $P$. Levy's sense. If the stable distribution is of index $\gamma$, the order of $\left\{x_{t}\right\}$ is $1 / \gamma$.

To prove this, notice that if the process is semi-stable of order $\alpha$ and $x_{0}=0$, then

$$
\phi_{t}(\lambda)=E\left(e^{i\left(\lambda, x_{t}\right)}\right)=E\left(e^{i t^{\alpha}\left(\lambda, x_{1}\right)}\right)=\phi_{1}\left(t^{\alpha} \lambda\right) .
$$

Since it also has stationary independent increments, we find that

$$
\phi_{1}\left(t^{\alpha} \lambda\right) \phi_{1}\left(s^{\alpha} \lambda\right)=\phi_{t}(\lambda) \phi_{s}(\lambda)=\phi_{t+8}(\lambda)=\phi_{1}\left[(t+s)^{\alpha} \lambda\right] .
$$

This, if $\alpha \neq 0$, implies that the distribution of $x_{1}$ (and so of all increments) satisfies (8) and so is stable in Levy's sense. The case $\alpha=0$ is trivial, for then all increments are 0 by Theorem 1 .

In one dimension the general form of a characteristic function which is 
stable of index $\gamma$ in the sense of (8) is as follows:

$$
\log \phi(t)=\left\{\begin{array}{lr}
-B|\lambda|^{\gamma}\left(1+i C \operatorname{sgn} \lambda \tan \frac{\pi \gamma}{2}\right), & 0<\gamma<2, \gamma \neq 1, \\
-B|\lambda|+i A \lambda, & \gamma=1, \\
-B \lambda^{2}, & \gamma=2 .
\end{array}\right.
$$

It is easily checked that each of these laws leads to an additive process which is semi-stable of order $1 / \gamma$, proving the converse part of the above statement when $s=1$. The same approach of direct verification, coupled with knowledge of the form of a stable law in several dimensions [6], suffices also for the multidimensional cases.

Example 2. Diffusion. We shall consider first a "classical" diffusion whose state space is a cone with vertex at the origin in $s$-dimensional space $\left({ }^{6}\right)$. That is, we assume that the backward equation is of the form

$$
\frac{\partial u}{\partial t}=\frac{1}{2} \sum_{i, j=1}^{s} a_{i j}\left(x^{(1)}, \cdots, x^{(s)}\right) \frac{\partial^{2} u}{\partial x^{i} \partial x^{j}}+\sum_{i} b_{i}\left(x^{(1)}, \cdots, x^{(s)}\right) \frac{\partial u}{\partial x^{(i)}},
$$

where the coefficients are given by

$$
\begin{aligned}
& b(\mathbf{x})=\lim _{h \rightarrow 0} E\left[\frac{\mathbf{x}_{t+h}-\mathbf{x}_{t}}{h} \mid \mathbf{x}_{t}=\mathbf{x}\right], \\
& \mathbf{a}(\mathbf{x})=\lim _{h \rightarrow 0} E\left[\frac{\left(\mathbf{x}_{t+h}-\mathbf{x}_{t}\right)^{\mathbf{T}}\left(\mathbf{x}_{t+h}-\mathbf{x}_{t}\right)}{h} \mid \mathbf{x}_{t}=\mathbf{x}\right] .
\end{aligned}
$$

(Here and below $b, x, x_{t}$, etc. are row vectors with components $b_{i}, x^{(i)}$, etc., $a$ is the $s \times s$ matrix $\left[a_{i j}\right]$, and the superscript $T$ means transpose.) It is under these assumptions quite easy to show that the functions $a(x)$ and $b(x)$ are arbitrary only on a sphere. More precisely, if the above diffusion is semi-stable of order $\alpha$ then

$$
a(x)=\|x\|^{2-1 / \alpha} a\left(\frac{x}{\|x\|}\right) ; \quad b(x)=\|x\|^{1-1 / \alpha} b\left(\frac{x}{\|x\|}\right) .
$$

To derive (11) we first write

$$
a(x)=\lim _{h \rightarrow 0} \frac{1}{h} \int_{y}(y-x)^{T}(y-x) d p_{h}(x, y),
$$

where $p_{t}(x, y)$ is the transition probability function of $\left\{x_{t}\right\}$. We now need the relation

$\left.{ }^{6}\right)$ If a process is semi-stable and $x_{0}=0$, the state space must be invariant under positive scalar multiplication. 


$$
p_{a t}(x, E)=p_{t}\left(a^{-\alpha} \mathbf{x}, a^{-\alpha} E\right) .
$$

This is immediate from the definition of semi-stable processes and Theorem 1, for $\left\{\boldsymbol{x}_{\boldsymbol{a}}\right\}$ and $\left\{a^{\alpha} \boldsymbol{x}_{t}\right\}$ are the same process (in the sense of finite-dimensional distributions) and so must have effectively identical transition functions. Now

$$
\boldsymbol{a}\left(a^{-\alpha} \mathbf{x}\right)=\lim _{h \rightarrow 0} \frac{1}{h} \int\left(\boldsymbol{y}-a^{-\alpha} \mathbf{x}\right)^{T}\left(\boldsymbol{y}-a^{-\alpha} \mathbf{x}\right) d p_{h}\left(a^{-\alpha} \mathbf{x}, \boldsymbol{y}\right),
$$

and with a change of variable and use of (12) we obtain

$$
\boldsymbol{a}\left(a^{-\alpha} \mathbf{x}\right)=\lim _{h \rightarrow 0} \frac{a^{1-2 \alpha}}{a h} \int(y-\mathbf{x})^{T}(y-\mathbf{x}) d p_{a h}(\mathbf{x}, \mathbf{y})=a^{1-2 \alpha} \mathbf{a}(\mathbf{x}) .
$$

Substitute $a^{\alpha}=\|x\|$ and this becomes the first part of (11); the second part is obtained in exactly the same way.

The result (11) does not, of course, establish the existence of semi-stable diffusion processes-especially since the origin is a singular point of (9) except in very special cases. A recent paper [4] studies convergence to these processes in the case when the state-space is the positive half-line; some results on the domain of attraction can be found there as well as a proof that convergence is weak $\left({ }^{2}\right)$ rather than simply in the sense of finite dimensional distributions. Even in this case the definition of $a$ and $b$ on the unit sphere-which reduces to choice of two constants - is not entirely arbitrary, and in many cases equation (9) must be supplemented by a reflecting barrier boundary condition. The higher-dimensional cases have not been analyzed; we only remark that $s$-dimensional Brownian motion is semi-stable of order $1 / 2$, and more generally any direct product of semi-stable processes of order $\alpha$ is itself semi-stable of order $\alpha$.

The one-dimensional case has been more completely studied by $\mathrm{C}$. Stone in his recent thesis [7]. Stone raises and solves a question which may be paraphrased as follows: When does a one-dimensional diffusion contain in its domain of attraction a diffusion or a birth and death process? By our Theorem 2, a process which does so is semi-stable. It is not quite obvious that every semistable diffusion is among the processes Stone finds, for he requires weak convergence and restricts the class of possible attractees. However, as the simple proof of the converse part of Theorem 2 (below) will show, any semi-stable diffusion does have a diffusion in its domain, namely itself, and for this choice of $\left\{X_{t}\right\}$ convergence takes place in the weak sense. Thus the results of [7] answer the first of our two questions entirely in this case. Stone considers the most general one-dimensional diffusion, so that the backward equation need not have the classical form (9) and (10). It turns out that these equations do hold for all semi-stable diffusions (except at the origin) so that (11) is always valid. Stone also determines all birth-and-death processes which can be attracted; we refer to [7] for further details. 
EXAmple 3. Purely discontinuous processes. Let us consider an s-dimensional Markov process, with $x_{0}=0$, such that the transition probability satisfies

$$
p_{t}(\mathbf{x}, E)=[1-q(\mathbf{x}) t] \Phi E(\mathbf{x})+t q(\mathbf{x}) P(\mathbf{x}, E)+o(t),
$$

where $\Phi_{E}$ is the characteristic function of $E . P(\mathbf{x}, E)$ is then a distribution which governs where a jump from $x$ will end, and $q(x)>0$ determines the holding time in state $x$. We shall not discuss here questions about the existence of such processes, but content ourselves with the remark that if a Markov process with transition function satisfying (13) is to be semi-stable of order $\alpha$, then it is necessary that

$$
q(\mathrm{x})=\|\mathrm{x}\|^{-1 / \alpha} q\left(\frac{\mathrm{x}}{\|\mathrm{x}\|}\right), \quad P(\mathrm{x}, E)=P\left(\frac{\mathrm{x}}{\|\mathrm{x}\|},\|\mathrm{x}\|-1 E\right) .
$$

The proof is similar to the argument above for diffusions. We combine (12) with (13) and obtain, for any $a>0$,

$$
q\left(a^{-\alpha} \mathbf{x}\right)=a q(\mathbf{x}), \quad P\left(a^{-\alpha} \mathbf{x}, a^{-\alpha} E\right)=P(\mathbf{x}, E)
$$

from which (14) follows.

EXAmple 4. Zeros of a semi-stable process. Suppose that $\left\{x_{t}\right\}$ is a measurable strong Markov process with $x_{0}=0$, and define

$$
y_{t}=t-\sup \left\{\tau \mid \tau \leqq t, x_{\tau}=0\right\} .
$$

Then $\left\{y_{t}\right\}$ is a Markov process whose paths consist of a collection of straightline segments of slope 1 with left ends on the $t$-axis, plus the remaining points of the axis itself. It is very easy to see that if $\left\{x_{t}\right\}$ is semi-stable of any order, $\left\{y_{t}\right\}$ is semi-stable of order one. If $\left\{x_{t}\right\}$ is chosen as one of the one-dimensional processes of Examples 1 or 2, a class of processes $\left\{y_{t}\right\}$ results which arose in a different way in [5]. The discussion there contains some information about the domains of attraction of these processes, and, more important for present purposes, the form of the transition probabilities is given. These are as follows:

$$
\begin{aligned}
p_{t}(y,\{y+t\}) & =\left(\frac{y}{y+t}\right)^{\lambda} \quad \text { for } y>0 \\
p_{t}(0,[0, y]) & =F_{1-\lambda}\left(\frac{y}{t}\right)=\frac{\sin \pi \lambda}{\pi} \int_{0}^{\min (1, y / t)} u^{-\lambda}(1-u)^{\lambda-1} d u .
\end{aligned}
$$

Here the $F_{1-\lambda}$ are "generalized arc-sine laws" and $\lambda$ is a parameter between 0 and $1\left(^{7}\right)$. It is clear that if the strong Markov property is assumed, $p_{t}(x,[0, y])$ can be calculated from (16) as follows:

( ${ }^{7}$ ) Only the cases $0<\lambda \leqq 1 / 2$ occur in Example 1, all of them in Example 2 [5]. Taking as $\left\{x_{t}\right\}$ the stable process of order $\gamma, 1<\gamma \leqq 2$, corresponds to the value $\lambda=1-1 / \gamma$ in (16). 


$$
p_{t}(x,[0, y])
$$

$$
=\left\{\begin{array}{l}
1 \quad \text { if } x+t \leqq y, \\
\int_{u=0}^{t} F_{1-\lambda}\left(\frac{y}{t-u}\right) d\left\{1-\left(\frac{x}{x+u}\right)^{\lambda}\right\} \quad \text { if } x+t>y, \text { for } x>0 .
\end{array}\right.
$$

We shall show that (16) and (17) are the only possible form for the transition probabilities of a process of this sort. The interest of this fact is perhaps greatest if it is borne in mind that we are essentially determining the stochastic structure of the $t$-set where $x_{t}=0$, where $\left\{x_{t}\right\}$ is any strongly Markovian semi-stable process. The conclusion can be stated more formally as follows:

THEOREM 3. If $\left\{x_{t}\right\}$ (with $x_{0}=0$ ) is a semi-stable measurable strong Markov process with stationary transition probabilities, and $\left\{y_{t}\right\}$, defined by (15), is a proper process, then the transition probabilities of $\left\{y_{t}\right\}$ are given by (16) and (17) for some $\lambda \in(0,1)$.

The proof, which is somewhat lengthy, is presented in the next section.

3. Proofs. We remark first regarding the theorems of $\$ 1$ that it is only necessary to consider the one dimensional case, since the arguments we give are easily extended to the case of vector-valued processes. In fact if a vector process is semi-stable, so is each component process; this is immediate from the definition. The function $b(a)$ is common to the $s$ component processes, so Theorem 1, if true when $s=1$, is immediate for any $s$. As for Theorem 2, choose a component process which is proper and use the one-dimensional proof to determine $f(\xi)$; the remaining statements follow easily.

Proof of Theorem 1. We first show that $b(a)$ and $c(a)$ are continuous functions of $a>0$ if $\left\{x_{t}\right\}$ is a proper process. Indeed, we have from the semi-stable property that

$$
\operatorname{Pr}\left(x_{a+h} \leqq y\right)=\operatorname{Pr}\left(x_{1} \leqq \frac{y-c(a+h)}{b(a+h)}\right) .
$$

From the assumed continuity of the process, the left side approaches

$$
\operatorname{Pr}\left(x_{a} \leqq y\right)=\operatorname{Pr}\left(x_{1} \leqq \frac{y-c(a)}{b(a)}\right)
$$

as $h \rightarrow 0$. Noting that the distribution of $x_{1}$ is nondegenerate $\left({ }^{8}\right)$ and applying the theorem on p. 42 of [1] we find that $b(a+h) \rightarrow b(a)$ and $c(a+h) \rightarrow c(a)$, the desired continuity.

(8) Actually, if $\left\{x_{t}\right\}$ is semi-stable, then $x_{t}$ is nondegenerate for all $t>0$ if it is nondegenerate for one such $t$. 
Next observe that again from the semi-stable property we have

$$
\operatorname{Pr}\left(x_{s t} \leqq y\right)=\operatorname{Pr}\left(x_{1} \leqq \frac{y-c(s t)}{b(s t)}\right),
$$

and at the same time

$$
\operatorname{Pr}\left(x_{s t} \leqq y\right)=\operatorname{Pr}\left(x_{s} \leqq \frac{y-c(t)}{b(t)}\right)=\operatorname{Pr}\left(x_{1} \leqq \frac{[y-c(t)] b(t)^{-1}-c(s)}{b(s)}\right) .
$$

Comparison yields, using again the nondegeneracy of the distribution of $x_{1}$,

$$
b(s) b(t)=b(s t) ; \quad c(s t)=c(t)+c(s) b(t)\left({ }^{9}\right) .
$$

Thus $b(t)=t^{\alpha}$ for some constant $\alpha$, and if $\alpha=0$, we also obtain $c(t)=A \log t$. If $\alpha \neq 0$, the functional equation for $c$ is most easily solved by the change of variable $t=e^{v}, s=e^{u}$. The function $d(u)=c(s)$ then satisfies

$$
d(u+v)=d(v)+d(u) e^{\alpha v}
$$

the general (continuous) solution is

$$
d(u)=D\left(1-e^{\alpha u}\right), \text { or } \quad c(s)=D\left(1-s^{\alpha}\right) .
$$

This result is proved in a familiar way by letting $D=d(1)\left(1-e^{\alpha}\right)$, and then using induction to verify (20) as $u$ varies over the positive integers. Another induction shows that (20) holds for $u=2^{-n}$. But if it holds for two numbers it holds for their sum, so (20) is correct for all positive dyadic rationals. Continuity and an appeal to (19) with $v=-u$ completes the verification.

To continue the proof of the theorem, suppose next that $\alpha=0$. We have then

$$
\operatorname{Pr}\left(x_{t} \leqq y\right)=\operatorname{Pr}\left(x_{1} \leqq y-A \log t\right) .
$$

As $t \rightarrow 0$ this must approach the distribution of $x_{0}$, which is impossible unless $A=0$. Substituting in the definition of the semi-stable property we see that $\operatorname{Pr}\left(x_{0} \leqq x, x_{t} \leqq y\right)$ is independent of $t$. If we once again let $t \rightarrow 0$, from the continuity assumption (1) we see that this joint distribution is such that $x_{t}=x_{0}$ a.s.

Next consider $\alpha>0$. Using the results above we have

$$
\operatorname{Pr}\left(x_{t} \leqq x\right)=\operatorname{Pr}\left(x_{1} \leqq \frac{x-D}{t^{\alpha}}+D\right)
$$

As $t \rightarrow 0, \operatorname{Pr}\left(x_{t} \leqq x\right)$ approaches the distribution of $x_{0}$ by (1). The right side of (21), however, becomes degenerate at $x=D$ so that $D$ must be the (neces$b=d$.

(9) If $F()$ is a nondegenerate distribution, $a \neq 0$, and $F(a y+b) \equiv F(c y+d)$, then $a=c$ and 
sarily nonrandom) initial state. Finally, if $\alpha<0$ and again $t \rightarrow 0$, (21) yields $\operatorname{Pr}\left(x_{1} \leqq D\right)$ as the distribution of $x_{0}$; since this is a constant rather than a distribution function, this case is impossible. This observation completes the proof of Theorem 1.

Proof of Theorem 2. We shall use the following

Lemma. Suppose that for some $a_{n}>0, \alpha_{n}>0$, and distributions $F_{n}, G_{i}$,

$$
G_{n}\left(a_{n} x+b_{n}\right) \rightarrow F_{1}(x) \quad \text { and } \quad G_{n}\left(\alpha_{n} x+\beta_{n}\right) \rightarrow F_{2}(x), \quad n \rightarrow \infty,
$$

in the sense of convergence of distribution functions, and that $F_{1}$ and $F_{2}$ are nondegenerate. Then

$$
0<\lim _{n \rightarrow \infty} \frac{a_{n}}{\alpha_{n}}<\infty \quad \text { and } \quad \lim _{n \rightarrow \infty} \frac{b_{n}-\beta_{n}}{a_{n}} \neq \pm \infty
$$

both exist.

Proof. This lemma is a slight extension of a theorem $[1$, p. 42] which supposes $F_{1}=F_{2}$ and concludes that $\alpha_{n} / a_{n} \rightarrow 1$ and $\left(b_{n}-\beta_{n}\right) / a_{n} \rightarrow 0$. The present case is easily reduced to that one. First note that by the theorem in $[1$, p. 40] the distributions $F_{1}$ and $F_{2}$ must be of the same type; that is,

$$
F_{2}(x)=F_{1}(p x+q)
$$

for some $p>0, q$. The second assumed limit can therefore be written as

$$
G_{n}\left(\alpha_{n} \frac{u-q}{p}+\beta_{n}\right) \rightarrow F_{1}(u)
$$

The theorem mentioned above then applies and yields

$$
\lim _{n \rightarrow \infty} \frac{\alpha_{n}}{p a_{n}}=1 ; \quad \lim _{n \rightarrow \infty} \frac{b_{n}-\left(\beta_{n}-\alpha_{n} \frac{q}{p}\right)}{a_{n}}=0
$$

from which the conclusion is immediate.

This result will now be used to demonstrate (6). To accomplish this, let $\left\{\xi_{n}\right\}$ be any sequence of positive numbers approaching $\infty$. Then by (5) (using only convergence of one-dimensional distributions) we have when $t=1$

$$
\operatorname{Pr}\left\{X_{\xi_{i}} \leqq f\left(\xi_{i}\right) x-g\left(\xi_{i}\right)\right\} \rightarrow \operatorname{Pr}\left(x_{1} \leqq x\right) .
$$

But also for any $t>0$, replacing $\xi$ by $\xi t$ and $t$ by $1 / t$ in (5) yields

$$
\operatorname{Pr}\left\{X_{\xi_{i}} \leqq f\left(\xi_{i} t\right) x-g\left(\xi_{i} t\right)\right\} \rightarrow \operatorname{Pr}\left(x_{t-1} \leqq x\right) .
$$

From the lemma and the assumption that $\left\{x_{t}\right\}$ is a proper process, this implies that for each $t>0$ 


$$
\lim _{i \rightarrow \infty} \frac{f\left(\xi_{i} t\right)}{f\left(\xi_{i}\right)}=b(t)
$$

exists; since there is a limit for every sequence $\xi_{i} \rightarrow \infty$ the limit holds simply as $\xi \rightarrow \infty$. But it is known [3] (and not difficult to prove) that the existence of this limit for all $t>0$ implies that

$$
f(\xi)=\xi^{\alpha} L(\xi), \quad L \text { slowly varying, }
$$

which is the first part of (6).

To obtain the second part, we shall use the convergence of the initial distribution, assumed as part of (5):

$$
\operatorname{Pr}\left\{X_{0} \leqq f(\xi) x-g(\xi)\right\} \rightarrow \operatorname{Pr}\left(x_{0} \leqq x\right) .
$$

Since it was also assumed that $f(\xi) \nearrow \infty$ (which implies $\alpha \geqq 0$ ), the distribution of $x_{0}$ must be degenerate at a point, say $\omega$. Then if $y$ is a point of increase of the distribution of $X_{0}$, we must have for any $\epsilon>0$,

$$
f(\xi)(\omega-\epsilon)-g(\xi) \leqq y-\epsilon<y+\epsilon \leqq f(\xi)(\omega+\epsilon)-g(\xi)
$$

for all large values of $\xi$. This implies that

$$
\lim _{\xi \rightarrow \infty} \frac{g(\xi)}{f(\xi)}=\omega=x_{0},
$$

which together with (22) yields (6). The function $g(\xi)$ thus can be neglected just when the process $\left\{x_{t}\right\}$ starts from 0 .

The rest of the proof is simple. We have assumed in (5) that

$$
\begin{aligned}
\lim _{\xi \rightarrow \infty} \operatorname{Pr}\left[\frac{X_{\xi t_{1}}+g(\xi)}{f(\xi)}\right. & \left.\leqq x_{1}, \cdots, \frac{X_{\xi t_{k}}+g(\xi)}{f(\xi)} \leqq x_{k}\right] \\
& =\operatorname{Pr}\left[x_{t_{1}} \leqq x_{1}, \cdots, x_{t_{k}} \leqq x_{k}\right]
\end{aligned}
$$

We also have

$$
\lim _{\xi \rightarrow \infty} \operatorname{Pr}\left[\left\{\frac{X_{a \xi t}}{f(\xi)}+\frac{g(\xi)}{f(\xi)} \leqq x\right\}\right]=\operatorname{Pr}\left[\left\{x_{a t} \leqq x\right\}\right],
$$

where the several variables $X_{a \xi t_{1}}, \cdots, X_{a \xi t_{k}}$, etc., are indicated symbolically by the braces. Using (22) and the definition of a slowly varying function, the left-hand side of (24) becomes

$$
\lim _{\xi \rightarrow \infty} \operatorname{Pr}\left[\left\{a^{\alpha} u_{1}(\xi) \frac{X_{a \xi t}}{f(a \xi)}+u_{2}(\xi) \omega \leqq x\right\}\right]
$$

where $u_{j}(\xi)$ is a function which approaches 1 as $\xi \rightarrow \infty$. When this is written in the form 


$$
\lim _{\xi \rightarrow \infty} \operatorname{Pr}\left[\left\{\frac{X_{a \xi t}}{f(a \xi)}+\omega \leqq a^{-\alpha}\left(x u_{3}(\xi)+\omega\left(1-u_{4}(\xi) a^{\alpha}\right)\right)\right\}\right],
$$

it is clear that at continuity points the limit must be

$$
\operatorname{Pr}\left[\left\{x_{t} \leqq a^{-\alpha}\left(x-\omega\left(1-a^{\alpha}\right)\right)\right\}\right]
$$

This shows that the relation

$$
\left\{x_{a t}\right\} \approx\left\{a^{\alpha} x_{t}+\omega\left(1-a^{\alpha}\right)\right\}
$$

holds, and the process $\left\{x_{t}\right\}$ is thus semi-stable of order $\alpha$ and with initial state $\omega$. Note that $\alpha=0$ is not possible when $f(\xi) \nearrow \infty$ unless the limit process is improper, since the initial distribution, and hence each distribution if $\alpha=0$, is degenerate.

The converse is trivial, for if $\left\{x_{t}\right\}$ is semi-stable of order $\alpha>0$, we can choose for $\left\{X_{t}\right\}$ the process $\left\{x_{t}\right\}$ itself. The function $f(\xi)$ can then be taken to be $\xi^{\alpha}$, and $g(\xi)^{\alpha}$ to be $\omega \xi^{\alpha}$. This gives

$$
\operatorname{Pr}\left[\left\{\frac{X_{\xi t}}{f(\xi)}+\frac{g(\xi)}{f(\xi)} \leqq x\right\}\right]=\operatorname{Pr}\left[\left\{\frac{\xi^{\alpha} x_{t}+\omega\left(1-\xi^{\alpha}\right)}{\xi^{\alpha}}+\omega \leqq x\right\}\right],
$$

and letting $\xi \rightarrow \infty$,

$$
\lim _{\xi \rightarrow \infty} \operatorname{Pr}\left[\left\{\frac{X_{\xi t}}{f(\xi)}+\frac{g(\xi)}{f(\xi)} \leqq x\right\}\right]=\operatorname{Pr}\left[\left\{x_{t} \leqq x\right\}\right] .
$$

This shows also that no additional semi-stable processes are obtained by allowing discrete-parameter processes in the role of $\left\{X_{t}\right\}$ beyond those arising when the approximating process has a continuous parameter.

Proof of Theorem 3. To begin with we shall show that the first part of (16) must hold. Under the hypotheses of the theorem $\left\{y_{t}\right\}$ is semi-stable of order 1 and $y_{0}=0$, so that

$$
p_{t}(x,\{x+t\})=p_{1}\left(\frac{x}{t},\left\{\frac{x}{t}+1\right\}\right)=q\left(\frac{x}{t}\right), \text { say. }
$$

By the Markov property, on the other hand, we have

$$
p_{t+s}(x,\{x+t+s\})=p_{t}(x,\{x+t\}) p_{s}(x+t,\{x+t+s\}) .
$$

This is true since the transition from $x$ to $x+t+s$ in time $t+s$ can only take place along a straight line $\left({ }^{10}\right)$. Translating this equation into one for the function $q$ yields

${ }^{(10)}$ It should be observed that because of the strong Markov property the transition probabilities are defined and satisfy the above relations for all, not merely almost all, values of $x$. 


$$
q\left(\frac{x}{t+s}\right)=q\left(\frac{x}{t}\right) q\left(\frac{x+t}{s}\right) \quad \text { for all } x, s, t>0
$$

Make the change of variable $q(u)=r(u /(u+1))$ and (27) becomes

$$
r\left(\frac{x}{x+t+s}\right)=r\left(\frac{x}{x+t}\right) r\left(\frac{x+t}{x+t+s}\right) .
$$

In other words, $r(a b)=r(a) r(b)$ for all $a, b \in(0,1)$. This relation can be extended to all positive numbers by defining $r(a)$ to be $r\left(a^{-1}\right)^{-1}$ when $a>1$; the function $r$ is certainly measurable. It is well known that this implies that $r(a)=a^{\lambda}$, which in turn means that

$$
p_{t}(x,\{x+t\})=q\left(\frac{x}{t}\right)=\left(\frac{x}{x+t}\right)^{\lambda} .
$$

Since this is decreasing in $t$ and $\left\{y_{t}\right\}$ is proper, $\lambda>0$.

Now, using the semi-stable property, let

$$
p_{t}(0,[0, y])=p_{1}\left(0,\left[0, \frac{y}{t}\right]\right)=F\left(\frac{y}{t}\right),
$$

where $F()$ is a distribution on the unit interval. The transition from 0 to $[0, y]$ in time 1 occurs if and only if the $\left\{y_{t}\right\}$ process attains the value zero for some $t \in[1-y, 1]$. By integrating over the possible states of the process at time $1-y$, we obtain for $y>0$

$$
F(y)=\int_{u=0}^{1-y}\left[1-p_{\nu}(u,\{u+y\})\right] d F\left(\frac{u}{1-y}\right)
$$

as a necessary condition which the unknown function $F$ must satisfy. We shall see that when $\lambda<1$ the function $F_{1-\lambda}$ of (16) is the only acceptable solution, and that there is none if $\lambda \geqq 1$.

To achieve this let us rewrite the necessary condition above as

$$
F(y)=\int_{0}^{1}\left[1-\left\{\frac{\omega(1-y)}{\omega(1-y)+y}\right\}^{\lambda}\right] d F(\omega) .
$$

Next, consider a transformation $T$ of measures on $(0,1]$ defined by

$$
T \mu(y, 1]=\int_{0}^{1} \frac{\omega^{\lambda}(1-y)^{\lambda}}{[\omega(1-y)+y]^{\lambda}} d \mu(\omega) .
$$

It is easily checked that $T \mu(y, 1]$ is decreasing in $y$, zero if $y=1$ and equal to $\mu(0,1]$ if $y=0$; in particular, if $\mu$ is a probability measure on $(0,1]$ so is $T \mu$. The condition (28) is equivalent to stating that the measure generated by 
$F(y)$ consists of a possible mass at 0 , plus a measure on $(0,1]$ which is a fixpoint of $T$.

The transformation $T$ can be used to generate a discrete-time Markov process $\left\{X_{n}\right\}$ on $(0,1]\left({ }^{11}\right)$. Indeed, if any (Borel) probability measure on $(0,1]$, say $\mu_{0}$, is taken as initial distribution then $T^{n} \mu_{0}$ is the distribution of $X_{n}$; the transition probabilities from a point $\xi$ are defined by applying $T$ to the measure concentrating unit mass at $\xi$. These transition probabilities have densities which are uniformly bounded from 0 provided $\xi$ is bounded from 0 . In view of this it is easy to see that, for each $\lambda>0$, one or the other of the following two possibilities must hold: (i) for any choice of $X_{0}$ there is a positive probability that $X_{n} \rightarrow 0$ as $n \rightarrow \infty$, or (ii) the process $\left\{X_{n}\right\}$ is recurrent in the sense that any set of positive (Lebesgue) measure is eventually entered with probability one from any initial state $(>0)$. It is clear that only in case (ii) can there be a probability measure on $(0,1]$ which is a fix-point of $T$.

We now appeal to a theorem of T. E. Harris [2] which says that in case (ii) there is a unique $\sigma$-finite measure which is invariant under $T$. Notice now that for every $\lambda>0$, the function

$$
\frac{d \mu(\omega)}{d \omega}=\omega^{-\lambda}(1-\omega)^{\lambda-1}
$$

is the density of an invariant measure on $(0,1]$; this measure is finite if $\lambda<1$, but infinite if $\lambda \geqq 1$. In the latter case, therefore, no probability measure on $(0,1]$ is a fix-point of $T$, and so the only distribution function satisfying (28) is $H(y)$, the function with a unit step at the origin. Recalling the role of this function in the transition probabilities of $\left\{y_{t}\right\}$, we see that if $\lambda \geqq 1$, $y_{t}=0$ a.s. for all $t$ and no proper process exists of the kind we are considering.

Turning to the case $\lambda<1$, we now see that the "arc-sine law" $F_{1-\lambda}$ (of which (29) is the density) is the only probability measure on $(0,1]$ which is invariant under $T\left({ }^{12}\right)$; hence

$$
p F_{1-\lambda}(y)+q H(y)=F(y), \quad p, q \geqq 0, p+q=1
$$

is the most general probability distribution on $[0,1]$ satisfying $(28)$. Notice that $\operatorname{Pr}\left(y_{t}=0\right)=q$ for all $t>0$. Now

$$
\operatorname{Pr}\left(y_{t+h}=0\right)=q=p \int_{0+}^{t} p_{h}(u,\{0\}) d F_{1-\lambda}\left(\frac{u}{t}\right)+a^{2} .
$$

But clearly

(11) This process is simply a technical device in the proof, and has no relation with the other processes studied in this paper.

(12) Since (29) gives a finite invariant measure, we must have case (ii) and Harris' theorem applies. 


$$
p_{h}(u,\{0\}) \leqq 1-p_{h}(u,\{u+h\})=1-\left(\frac{u}{u+h}\right)^{\lambda},
$$

and so the integral in (30) tends to 0 as $h \rightarrow 0$ by dominated convergence. This leaves us with $q=q^{2}$. The case $q=1$ gives again an improper process $\left\{y_{t}\right\}$, so $q=0$ and the proof that (16), and hence also (17), must hold is complete.

4. Gaussian and "wide sense" processes. Suppose that $\left\{x_{t}\right\}$ is a real Gaussian process with mean 0 ; the continuity condition (1) then implies that the covariance function

$$
\rho(s, t)=E\left(x_{8} x_{t}\right)
$$

is continuous. We shall now show that the process $\left\{x_{t}\right\}$ is semi-stable of order $\alpha>0$ if and only if there is a nondecreasing function $F(\xi)$ of bounded variation such that

$$
\rho(s, t)=(s t)^{\alpha} \int_{0}^{\infty} \cos \left(\xi \log \frac{s}{t}\right) d F(\xi)
$$

To see this, recall from $\S 1$ that if $\left\{x_{t}\right\}$ is semi-stable of order $\alpha>0$, then $\left\{y_{\tau}=e^{-\alpha \tau} x_{e^{\tau}}\right\}$ is strictly stationary. The covariance function of $\left\{y_{\tau}\right\}$ is therefore of the form

$$
E\left(y_{r} y_{\sigma}\right)=\int_{0}^{\infty} \cos (\sigma-\tau) \xi d F(\xi)
$$

with $F()$ as described above; this translates into formula (31) for the covariance of $\left\{x_{t}\right\}$. Conversely, if a real Gaussian process with mean 0 has covariance function satisfying (as does (31)) the condition

$$
\rho(a s, a t)=a^{2 \alpha} \rho(s, t),
$$

it must be semi-stable. Indeed, from this condition we obtain

$$
E\left\{\left(\begin{array}{c}
x_{a t_{1}} \\
\vdots \\
x_{a t_{n}}
\end{array}\right)\left(x_{a t_{1}}, \cdots, x_{a t_{n}}\right)\right\}=E\left\{\left(\begin{array}{c}
a^{\alpha} x_{t_{1}} \\
\vdots \\
a^{\alpha} x_{t_{n}}
\end{array}\right)\left(a^{\alpha} x_{t_{1}}, \cdots, a^{\alpha} x_{t_{n}}\right)\right\}
$$

for any finite set $t_{1}, \cdots, t_{n}$. Since means and covariances determine multivariate normal laws, this implies that $\left(x_{a t_{1}}, \cdots, x_{a t_{n}}\right)$ has the same joint distribution as $\left(a^{\alpha} x_{t_{1}}, \cdots, a^{\alpha} x_{t_{n}}\right)$, so $\left\{x_{t}\right\}$ satisfies the condition of Definition 2.

It is of some interest to observe that a nontrivial semi-stable Gaussian Markov process with stationary transition probability and zero means must be, to within a scale factor, the Brownian motion process. If $\left\{x_{t}\right\}$ is Markovian, Gaussian, and semi-stable of order $\alpha$, the stationary process $\left\{y_{\tau}\right\}$ defined by (7) is a stationary Gaussian Markov process, and must accordingly (see 
[8], for instance) have covariance function

$$
E\left(y_{\tau} y_{\sigma}\right)=A e^{-B|\tau-\sigma|}, \quad A, B>0 ;
$$

the covariance of $\left\{x_{t}\right\}$ is therefore

$$
E\left(x_{s} x_{t}\right)=(s t)^{\alpha} A\left(\frac{s}{t}\right)^{B},
$$

But this is precisely the covariance function of

$$
z_{t}=A^{1 / 2} t^{\alpha-B} w_{t^{2 B}}
$$

where $\left\{w_{t}\right\}$ is Brownian motion; since the covariance determines the process $\left\{z_{t}\right\} \approx\left\{x_{t}\right\}$. It only remains to see that this process does not have stationary transition probabilities unless $2 B=1$ and $\alpha-B=0$; that is, unless it is (scaled) Brownian motion. Since $\left\{x_{t}\right\}$ is a Gaussian process, the transition probabilities are determined by the covariances, and in particular for $s \leqq t$,

$$
E\left(x_{t}^{2} \mid x_{s}=0\right)=A t^{2 \alpha}\left[1-\left(\frac{s}{t}\right)^{2 B}\right] .
$$

It is not difficult to see that this is a function of $t-s$ alone only in the case $\alpha=B=1 / 2$; q.e.d.

To conclude, we shall briefly discuss the "wide-sense" versions of the ideas of this paper, in the spirit in which the term is used in Doob's treatise [8]. We consider (not necessarily Gaussian) processes $\left\{x_{t}\right\}$ with $x_{0}=0$ and continuous covariance functions, and call such a process semi-stable in the wide sense (s.s.w.s.) if there is a function $b(a)>0$ such that

$$
E\left(x_{a s} x_{a t}\right)=E\left(b(a) x_{s} b(a) x_{t}\right) .
$$

We say that another process $\left\{X_{t}\right\}$, also with continuous covariance, is attracted in the wide sense to $\left\{x_{t}\right\}$ if there is a function $f(\xi) / \infty$ such that

$$
\lim _{\xi \rightarrow \infty} E\left[\frac{X_{\xi}}{f(\xi)} \frac{X_{\xi t}}{f(\xi)}\right]=E\left(x_{s} x_{t}\right) .
$$

This situation can be investigated by constructing Gaussian processes with mean 0 and covariance functions equal to those of $\left\{x_{t}\right\}$ and $\left\{X_{t}\right\}$, and applying our previous results for "strict-sense" semi-stable processes to these. In this way it is easy to establish the following facts: $A$ process $\left\{x_{t}\right\}$ with $x_{0}=0$ (but not $x_{t}=0$ for all $t$ ) is s.s.w.s. if and only if there exists a process $\left\{X_{t}\right\}$ which is attracted to it in the wide sense. If this is the case, there exists an $\alpha>0$ and a slowly varying function $L$ such that $b(a)=a^{\alpha}$ and $f(\xi)=\xi^{\alpha} L(\xi)$. Also, the process $\left\{x_{t}\right\}$ is s.s.w.s., if and only if there is an $\alpha>0$ and a bounded nondecreasing function $F$ such that $E\left(x_{s} x_{t}\right)$ is given by (31). 


\section{REFERENCES}

1. B. V. Gnedenko and A. N. Kolmogorov, Limit distributions for sums of independent random variables, Addison-Wesley, Cambridge, Mass., 1954 (translation by K. L. Chung).

2. T. E. Harris, The existence of stationary measures for certain Markov processes, Proc. 3rd Berkeley sympos., Vol. II, pp. 113-124, Berkeley, 1956. $55-62$.

3. J. Karamata, Sur un mode de croissance régulière, Bull. Soc. Math. France 61 (1933),

4. J. W. Lamperti, A new class of probability limit theorems, J. Math. Mech. 11 (1962), no. 5. An announcement of the main results is contained in Bull. Amer. Math. Soc. 67 (1961), 267269.

5. - An invariance principle in renewal theory, Ann. Math. Statist. 33 (1962), no. 2.

6. P. Levy, Theorie de l'addition des variables aleatoires, Gauthier-Villers, Paris, 1937.

7. C. J. Stone, Limit theorems for birth and death processes and diffusion processes, Stanford University thesis, June 1961.

8. J. L. Doob, Stochastic processes, Wiley, New York, 1953.

STANFORD University,

Stanford, California 